"C 2019 IEEE. Personal use of this material is permitted. Permission from IEEE must be obtained for all other uses, in any current or future media, including reprinting/republishing this material for advertising or promotional purposes, creating new collective works, for resale or redistribution to servers or lists, or reuse of any copyrighted component of this work in other works." 


\title{
Electrically Small Huygens CP Rectenna with a Driven Loop Element Maximizes Its Wireless Power Transfer Efficiency
}

\author{
Wei Lin, Member, IEEE and Richard W. Ziolkowski, Fellow, IEEE
}

\begin{abstract}
An electrically small Huygens circularly polarized (HCP) rectenna whose antenna is directly matched to its rectifying circuit is developed and experimentally validated in the ISM band at $915 \mathrm{MHz}$. The HCP antenna is a near-field resonant parasitic (NFRP) design consisting of a driven element and a crossed pair of balanced electric and magnetic NFRP dipole elements. The rectifier circuit is highly capacitive. It is a full-wave design based on two HSMS286C Schottky diodes. Two innovative driven element designs are explored that facilitate the effective conjoin of the antenna to different classes of external circuits. An HCP antenna with a driven spiral line element is demonstrated. Its input impedance is capacitive and, hence, the system is ideal for matching directly to an inductive external circuit. An HCP antenna with a driven loop element is investigated in a similar manner. The system has an inductive input impedance that is subsequently optimized to be conjugately match to the capacitive rectifier directly. The measured HCP rectenna prototype has broad-angle capture capacity, avoids any polarization mismatch issues, and has exceptional AC to DC conversion efficiency, the maximum reaching $\mathbf{9 0 . 6 \%}$. It is an ideal candidate for wireless power transfer (WPT)-enabled Internet-of-Things (IoT) applications.
\end{abstract}

Index Terms-Circular polarization, electrically small antennas, Huygens radiation patterns, Internet-of-Things (IoT), rectenna, wireless power transfer

\section{INTRODUCTION}

Far-field wireless power transfer (WPT) technology is an emerging trend for many expected wireless Internet-of-Things (IoT) applications [1] - [4]. The number of wireless IoT devices will witness exponential growth in the near future [5]. Since it will not be possible to replace the bulky and short-life batteries for all IoT devices manually, such as sensors that are embedded inside objects like bridges, pipes or sealed storage containers [6] - [8], WPT is an attractive solution for powering them. Moreover, current wire-powered sensor systems have major issues such as cable corrosion and difficulties in cable relocation [9], [10]. The rectenna is the key component in any far-field WPT system; it captures the electromagnetic waves and converts their AC power into DC power [11] - [13]. The user-friendly features of IoT systems will require their WPT rectennas to have compact sizes, high AC to DC conversion efficiencies, broad-angle wireless power capture capacities, and the capability to mitigate polarization mismatch issues in multipath environments.

Extensive efforts have been expended on the design of rectennas in recent years. However, it remains very challenging to fulfill all of the noted physical and performance criteria. The majority of the reported rectennas have been based on linearly polarized (LP) antenna systems. Unfortunately, they encounter polarization mismatch issues in most practical applications [14] - [23]. To mitigate this problem, a few works have reported dual-pol [24], [25] or circularly polarized (CP) [26] - [30] rectennas. However, none of those systems simultaneously have an electrically small size (i.e., have $k a<1$, where $k$ is the free space wave number and $a$ is the radius of the smallest sphere that encloses the entire system), a high AC to DC power conversion efficiency (near $90 \%$ ), and a large wireless power capture capacity (i.e., a broad half-power beamwidth $>120^{\circ}$ and an antenna gain $>3 \mathrm{dBic}$ ).

Manuscript received on Mar. 26, 2019
Wei Lin and Richard. W. Ziolkowski are with the University of Technology Sydney, Global Big Data Technologies Centre, School of Electrical and Data Engineering, Ultimo NSW 2007, Australia (E-mail: wei.lin@uts.edu.au).

To the best of our knowledge, only one system has achieved all of the indicated characteristics to date. It was the electrically small Huygens CP (HCP) rectenna reported in our previous work [31]. The antenna seamlessly integrates a driven unbalanced dipole element with a crossed pair of orthogonally-oriented balanced electric (Egyptian axe dipole, EAD) and magnetic (capacitively loaded loop, CLL) near-field resonant parasitic (NFRP) dipole elements. The HCP rectenna was achieved by connecting this NFRP antenna to a rectifier. Nevertheless, the overall system did not reach its maximum achievable AC to DC conversion efficiency because of its driven $50 \Omega$ unbalanced dipole structure. In particular, an inductor had to be introduced between its HCP antenna and its highly capacitive rectifying circuit to achieve a good impedance match. Unfortunately, this lumped component caused approximately a $7 \%$ conversion efficiency loss for the entire system. Considering the ubiquitous nature of future IoT devices and the associated large number of their rectennas, this loss corresponds to a significant waste of power simply to heating the environment.

In this communication, we report two innovative driven element structures, a spiral line-based and a loop line-based element. They facilitate connecting an HCP antenna directly to an external circuit without any intervening lumped elements for impedance matching. As will be discussed, the spiral line-based HCP antenna has a capacitive impedance, making it ideal for integration with inductive circuits. On the other hand, the loop line-based HCP antenna is inductive and ideal for integration with capacitive circuits. Consequently, the loop line-based HCP rectenna was advanced for the rectenna system. Its inductive impedance was optimized to yield a HCP antenna system that is conjugately matched to a highly capacitive, full-wave rectifying circuit based on HSMS286C Schottky diodes. A prototype HCP rectenna was fabricated and tested. It is electrically small with $k a$ 0.77 . It has a measured large antenna gain $(>3 \mathrm{dBic})$ and broad half-power beamwidth $\left(>130^{\circ}\right)$, indicative of a high capacity to capture any incident power. The measured peak AC to DC conversion efficiency reached $90.6 \%$, which is the maximum achievable and highest value obtained among those reported in a survey of rectennas operating in the $915 \mathrm{MHz}$ ISM band [32]. Further, there are no polarization mismatch issues because it is a CP system.

This communications is organized as follows. Sec. II discusses the two new driven line elementapproaches for the HCP NFRP antenna. Sec. III presents the design, fabrication and measurement of the optimizedprototype system. Sec. IV is the conclusion. The antenna simulations (impedance and radiation performance) were performed with the ANSYS Electromagnetics Suite 16.2. With the resulting two-port impedance representation of the antenna, the rectifying circuit was then co-designed and simulated with the Agilent advanced design system (ADS). The power flow (streamlines) plots (Fig. 9) were obtained with CST Microwave Studio.

\section{Electrically Small Huygens CP Antennas}

The HCP antenna reported in our previous work [33] was excited with an unbalanced cross dipole structure. It had a $50 \Omega$ input impedance at the frequency at which the axial ratio (AR) value was a 
minimum. When attempting to connect it directly to a rectifier, it was found to be difficult to maintain good $\mathrm{CP}$ performance while attempting to attain a significant inductive or a capacitive input impedance. It was determined that either goal could be achieved if the HCP's driven dipole structure was modified appropriately. The spiral line-based and the loop line-based driven elements were developed for this purpose. They facilitate the direct matching of the resulting HCP antennas, respectively, to inductive and capacitive circuits while still maintaining their $\mathrm{CP}$ performance without any additional impedance matching components.

\section{A. Driven spiral line structure}

The spiral line-based driven element shown in Fig. 1was explored initially. This concept arose from the fact that a two-arm spiral radiator naturally produces CP fields. To be integrated with the HCP system, it had to be designed to properly excite the indicated four electric and magnetic dipole NFRP elements: EAD\#1, EAD\#2, CLL\#1 and CLL\#2. An idealized voltage source was used in the simulation model. EAD\#1 (EAD\#2) and CLL\#1 (CLL\#2) are parallel and aligned along the $\mathrm{x}$-axis (y-axis). The spiral structure directly excites the crossed CLL elements. Because they are strongly coupled to the crossed EAD elements, they induce currents on them. The spiral line structure successfully achieved the CP target of exciting the two pairs of NFRP elements with the required $90^{\circ}$ phase difference. The current distributions on the bottom strips of the crossed CLL structure and the spiral line structure, as viewed from beneath them, are plotted in Fig. 2 at four different quarter-period times when the HCP antenna is driven at $915 \mathrm{MHz}\left(\lambda_{0}=327.64 \mathrm{~mm}\right)$. One clearly sees that the direction of the currents on these bottom strips is changing clockwise as time increases. Thus, since the field is propagating away from the viewer and the current rotation agrees with the right-handed rule, the dominant polarization is right-hand CP (RHCP). Moreover, the two orthogonal CLLs are seen to be resonating alternately with a quarter period difference, i.e., they have the noted $90^{\circ}$ phase difference.

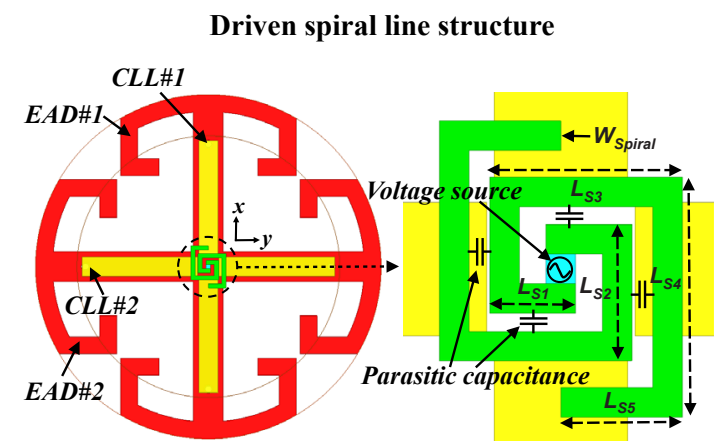

Fig. 1.The spiral line-based HCP antenna. (Left) Bottom view of the antenna and (Right) enlarged view of the driven spiral line element.

When one looks at the enlarged views of the currents on the spiral feeding structure carefully, it becomes obvious that the currents exhibit a travelling-wave behavior along the spiral arm. For example, the currents on the inner loop remain unchanged from Figs. 2(a) to2(b) while those on the outer loop changed their directions. The same behavior occurs from Figs. 2(c) to 2(d). This travelling-wave behavior produces the requisite $90^{\circ}$ phase difference. The detailed parameter values of the spiral structure are (in millimeters): $W_{\text {Spiral }}=1.0, L_{S I}=2.9$, $L_{S 2}=4.6, L_{S 3}=6.5, L_{S 4}=8.1, L_{S 5}=4.1$. The feed gap of the two spiral arms in the HFSS model was $1.0 \mathrm{~mm}$.

The simulated performance characteristics are shown in Fig. 3. They include the input impedance and AR values as functions of the source frequency, and the two-dimensional (2D) realized gain patterns at $915 \mathrm{MHz}$. Note that the impedance exhibits a capacitive value as shown in Fig. 3(a). This feature arises from the parasitic capacitances between the two spiral arms. The $\mathrm{AR}$ value is $2.2 \mathrm{~dB}$ and the impedance is 93 - j59 $\Omega$ at $915 \mathrm{MHz}$. Dominant Huygens (cardioid)RHCP radiation patterns are observed with their peak realized gain value being $3.3 \mathrm{dBic}$ and with a wide $134^{\circ}$ half-power (3-dB) beamwidth (HPBW), from $\pm 67^{\circ}$. All AR values are less than 3 $\mathrm{dB}$ within the HPBW of the antenna. The patterns in the two vertical planes, $\varphi=0^{\circ}$ and $\varphi=90^{\circ}$, are almost identical with their maxima in the broadside direction. The radiation efficiency (RE) and front-to-back-ratio (FTBR) values at $915 \mathrm{MHz}$ are $77.2 \%$ and $29.3 \mathrm{~dB}$. Nearly perfect cancellation of the back radiation is observed. Alternately, LHCP radiation is readily obtained simply if the spiral structure is switched to a counterclockwise orientation. The capacitive impedance of this design could be directly matched to the double current rectifier developed in [34], which has an inductive impedance.
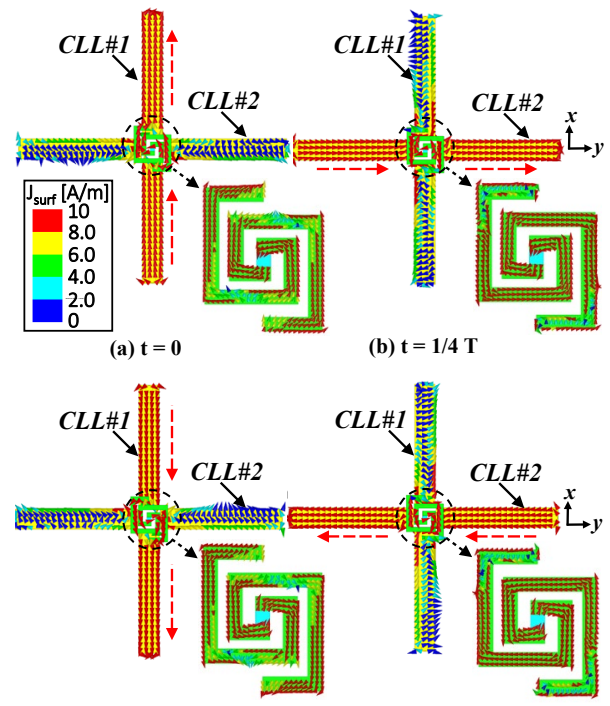

(c) $\mathrm{t}=2 / 4 \mathrm{~T}$

(d) $\mathrm{t}=3 / 4 \mathrm{~T}$

Fig. 2. Current distributions on the bottom strips of the crossed CLL elements and on the driven spiral line structure at four different quarter-period times of the $915 \mathrm{MHz}$ source frequency as viewed from underneath the HCP antenna.

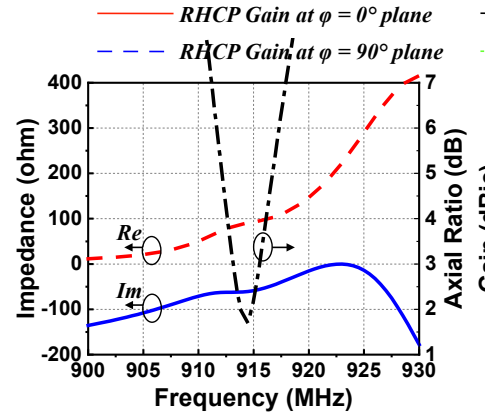

(a)

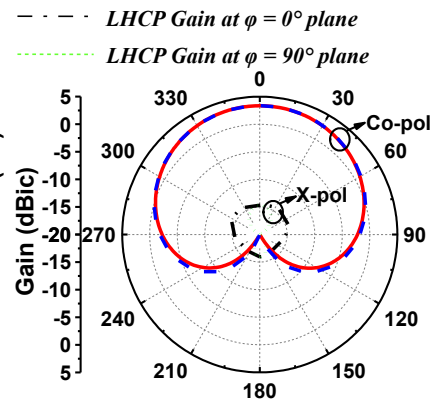

(b)
Fig. 3. Simulated results of the spiral-line-based HCP antenna. (a) Input impedance and AR values as functions of the source frequency. (b) The LHCP and RHCP realized gain patterns at $915 \mathrm{MHz}$.

\section{B. Driven loop structure}

The second excitation structure is the driven loop element shown in Fig. 4. It was inspired by the crossed-dipole CP antennas previously reported in [35] - [37]. The two dipoles are the indicated pair of two parallel straight arms. The two loops produce the required $90^{\circ}$ phase difference. When this structure is driven, it directly excites the currents 
on the bottom four strips of the crossed CLL elements. Fig. 5 shows the view of these current distributions and those on the excitation structure from beneath the antenna. Clockwise rotating currents are clearly seen again on these bottom strips and those on CLL\#1 and CLL\#2 are resonating alternately with a quarter-period interval, indicating the radiated field is RHCP. The current distributions on the loop structure also confirm the CP behavior. A travelling-wave behavior is also found on this loop structure. For example, the direction of the horizontal currents (along the y-axis) remain unchanged from Figs. 5(a) to 5(b), but their amplitudes change from large to small values. In contrast, the vertical currents (along the $\mathrm{x}$-axis) also maintain their directions, but are small at $t=0$ and then become strong at $t=T / 4$. The same behavior is observed over the quarter-period going from Figs. 5(c) to 5(d). These features reaffirm the requisite $90^{\circ}$ phase difference between the orthogonal NFRP elements.

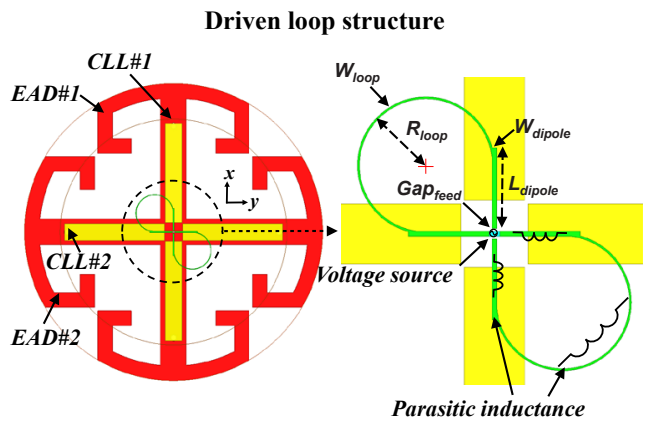

Fig. 4.The driven loop-based HCP antenna. (Left) Bottom view of the antenna and (Right) enlarged view of the driven loop element.
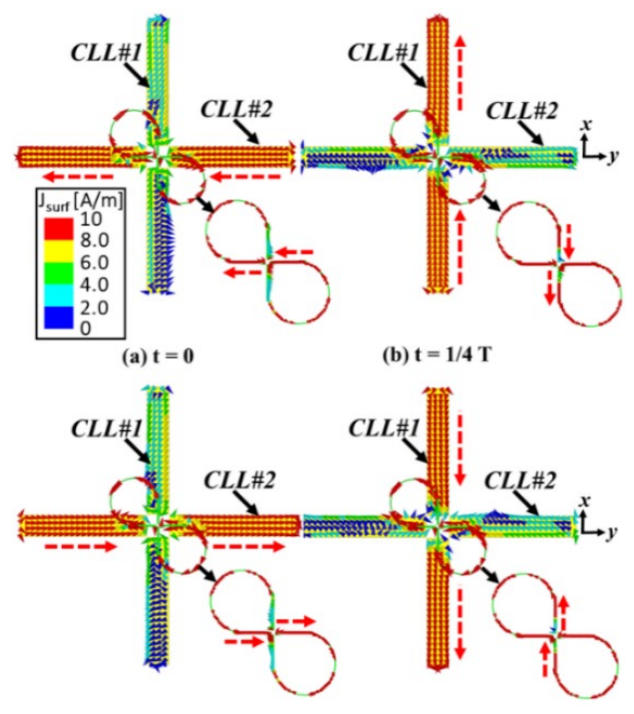

(c) $\mathrm{t}=\mathbf{2} / \mathbf{4} \mathrm{T}$

(d) $\mathrm{t}=3 / 4 \mathrm{~T}$

Fig. 5. Current distributions on the bottom strips of the crossed CLL elements and on the driven loop structure at four different quarter-period times of the 915 $\mathrm{MHz}$ source frequency as viewed from underneath the HCP antenna.

The simulated performance results are shown in Fig. 6. They include the input impedance and AR values as functions of the source frequency, and the $2 \mathrm{D}$ realized gain patterns at $915 \mathrm{MHz}$. The AR value is $1.7 \mathrm{~dB}$ and the input impedance is $57+\mathrm{j} 164 \Omega$ at $915 \mathrm{MHz}$. Dominant Huygens (cardioid) RHCP radiation patterns are observed with the peak gain value being $3.2 \mathrm{dBic}$ and with a wide $132^{\circ} \mathrm{HPBW}$, from $\pm 66^{\circ}$. All AR values are less than $3 \mathrm{~dB}$ within the HPBW of the antenna. The patterns in the two vertical planes, $\varphi=0^{\circ}$ and $\varphi=90^{\circ}$, are almost identical with their maxima in the broadside direction. The RE and FTBR values at $915 \mathrm{MHz}$ are $73.1 \%$ and $29 \mathrm{~dB}$. Nearly perfect cancellation of the back radiation is observed. Alternately, LHCP radiation can be obtained simply by changing the directly driven dipole from the $y$-axis element to the $x$-axis one. Since the feeding structure is completely symmetric with respect to its center axis, all of the other radiation characteristics will remain the same.

The characteristics of the HCP antenna with the two distinct driven structures are summarized in Table I. In contrast to the large capacitance associated with the HCP antenna with the driven spiral line structure, the simulated input impedance results of the HCP antenna with the driven loop element shown in Fig. 6(a) indicate that it exhibits a large input inductance. This feature occurs because the straight and loop strips of the driven element are quite long, creating the strong parasitic inductances depicted in Fig. 4 (Right subplot). With its high inductive input reactance, this HCP antenna is quite suitable for being directly matched to the highly capacitive rectifier circuit. Note that the capacitance of the highly efficient AC to DC convertor designed in [31] was around $-150 \Omega$. The HCP antenna with this driven loop element can be optimized to conjugately match it to that rectifier, eliminating the lossy inductor used in [31] for impedance matching.

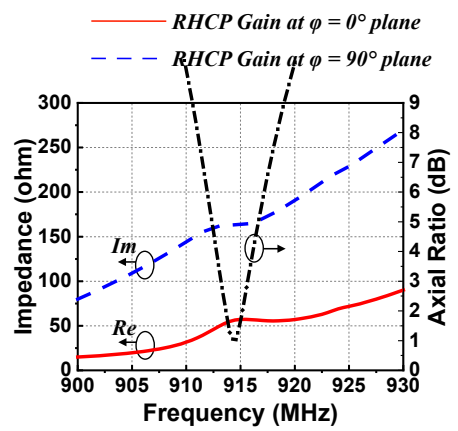

(a)

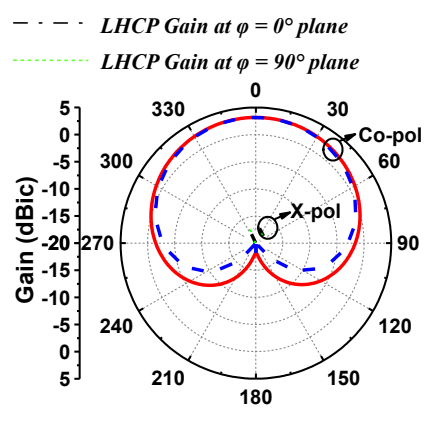

(b)
Fig. 6. Simulated results of the driven loop-based HCP antenna. (a) Input impedance and AR values as functions of the source frequency. (b) The LHCP and RHCP realized gain patterns at $915 \mathrm{MHz}$.

TABLE I:

ChaRACTERISTICS OF THE HCP ANTENNA WITH THE Two DistinCt DRIVEN STRUCTURES (SOURCE FREQUENCY: $915 \mathrm{MHz}$ )

\begin{tabular}{c|c|c|c|c}
\hline \hline $\begin{array}{c}\text { Driven } \\
\text { Structures }\end{array}$ & Impedance & Axial Ratio & Peak Gain & HPBW \\
\hline $\begin{array}{c}\text { Spiral } \\
\text { Line }\end{array}$ & $\begin{array}{c}93-\mathrm{j} 59 \Omega \\
(\text { Capacitive) }\end{array}$ & $2.2 \mathrm{~dB}$ & $3.3 \mathrm{dBic}$ & $\pm 67^{\circ}$ \\
\hline Loop & $\begin{array}{c}57+\mathrm{j} 164 \Omega \\
\text { (Inductive) }\end{array}$ & $1.7 \mathrm{~dB}$ & $3.2 \mathrm{dBic}$ & $\pm 66^{\circ}$ \\
\hline \hline
\end{tabular}

\section{Key design considerations}

The design parameters of the optimized system are listed in Table I. The radius, $R_{\text {loop }}$, and the width of the loop, $W_{\text {loop}}$, are critical design parameters in the driven loop element. Fig. 7gives the AR values as functions of the source frequency for different radius and width values. It is observed clearly in Fig.7(a) that both the lowest AR value and its frequency location vary noticeably when the loop radius $R_{\text {loop }}$ changes from 4.5 to $5.5 \mathrm{~mm}$ in $0.25 \mathrm{~mm}$ steps (keeping all other parameters fixed). The $R_{\text {loop }}=5 \mathrm{~mm}\left(0.015 \lambda_{0}\right)$ was selected in the final design as it facilitates the best CP radiation. Fig. 7(b) shows the AR values when the loop width $W_{\text {loop }}$ changes from 0.05 to $0.25 \mathrm{~mm}$ in $0.05 \mathrm{~mm}$ steps. Although the minimum AR values do not change much, the associated frequency point noticeably shifts. To meet the target frequency at 915 $\mathrm{MHz}$, the value $W_{\text {loop }}=0.15 \mathrm{~mm}$ was selected for the final design. The parameter studies also indicate that the width of the loop can be 
adjusted for fine tuning the $\mathrm{CP}$ operational frequency.

TABLE II:

HCP ANTENNA DESIGN PARAMETERS (DIMENSIONS IN MILLIMETERS)

\begin{tabular}{lll}
\hline \hline \multirow{2}{*}{ Parameter } & \multicolumn{1}{c}{ Description } & Value \\
& & \\
\hline$D_{C L L}$ & Diameter of Substrate\#1 and \#3 & 61.4 \\
$D_{E A D}$ & Diameter of the whole system & 80.8 \\
$H$ & Height of the crossed CLL elements & 12.8 \\
$h$ & Distance between Substrate\#2 and \#3 & 7.6 \\
$d$ & Diameter of the copper rod & 1.5 \\
$l_{1}$ & Length of substrate\#4 in z axis & 10.0 \\
$l_{2}$ & Length of substrate\#4 in y axis & 20.0 \\
$R_{\text {loop }}$ & Radius of the loop & 5.0 \\
$W_{\text {loop }}$ & Width of the loop & 0.15 \\
Gap Feed & Gap between the two loop arms & 0.80 \\
$L_{\text {dipole }}$ & Length of the straight driven part & 6.0 \\
$W_{\text {dipole }}$ & Width of the straight driven part & 0.36 \\
\hline \hline
\end{tabular}

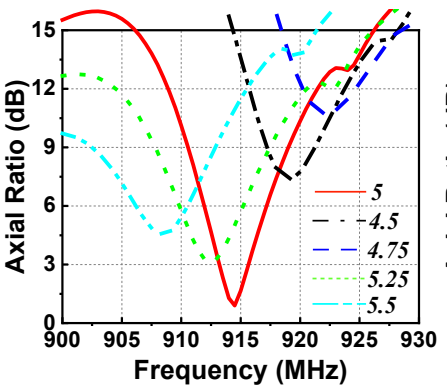

(a)

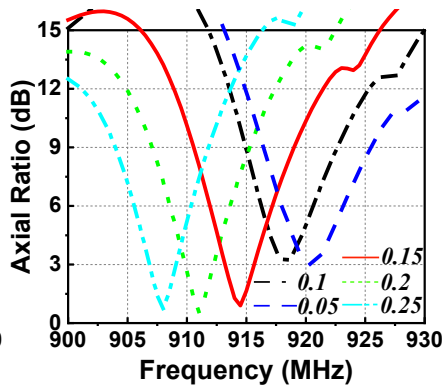

(b)
Fig. 7. Simulated AR values of the driven loop-based HCP antenna as functions of the source frequency for different design parameters (in millimeters).(a) Loop radius $R_{\text {loop }}$. (b) Loop width $W_{\text {loop }}$.

\section{Realization and Performance of the Electrically Small DRIVEN LOOP-BASED HCP RECTENNA}

\section{A. System Configuration}

The HCP antenna with the driven loop element was seamlessly integrated with a highly efficient rectifier circuit as shown in Fig. 8. The entire system consists of four substrates. The Huygens CP antenna consists of $S u b \# 1$ to $S u b \# 3$, located from top to bottom. The top and bottom metallization layers of the crossed CLL elements are etched on the top surface of $S u b \# 1$ and $S u b \# 3$, respectively. These two layers are connected by four vertical copper rods, each with a $1.5 \mathrm{~mm}$ diameter. The EAD element metallization layer is etched on the top surface of $S u b \# 2$, which is supported by a lossless Rohecell ${ }^{\mathrm{TM}}$ foam whose relative permittivity is 1.05 . The four copper rods go through the EAD layer with $0.25 \mathrm{~mm}$ annular isolation. The metallization layer of the driven loop element is located on the bottom of Sub\#3. The rectifier circuit is printed on the positive $x$-axis surface of $S u b \# 4$. The Sub\#4 is centered on $S u b \# 3$ with respect to the y-axis and is oriented vertically with respect to it. The input port of the rectifier is soldered to the port of the driven loop. All of the substrates are Rogers ${ }^{\mathrm{TM}} 5880$ copper-cladded $(0.017 \mathrm{~mm}$ thickness $)$ materials whose relative permittivity, permeability and loss tangent are equal to $2.2,1.0$ and 0.0009 , respectively. The thickness ofSub\#1, Sub\#3 and Sub\#4 is $0.7874 \mathrm{~mm}$ and that of $S u b \# 2$ is $0.508 \mathrm{~mm}$. The entire system is highly compact and electrically small at $915 \mathrm{MHz}$ with $k a \sim 0.77$.

The circuit model of the rectifier is shown in Fig. 8(c). It is a full-wave design based on two HSMS286C Schottky diodes [38]. These diodes are very suitable for the WPT application since their operational parameters facilitate a higher AC to DC conversion efficiency (near 90\%) at a higher input power level (near $10 \mathrm{dBm}$ ) when compared with other reported rectifiers [39] - [42]. Note that no lumped inductor is used in the circuit. The capacitors $C_{I}$ and $C_{2}$ are for impedance matching and are almost lossless. $C_{2}$ also acts as the energy storage component during each negative portion of the sinusoidal signal. $C_{3}$ works as a smoothing filter that minimizes the ripples in the output DC voltage. $R_{L}$ is the load resistor. To facilitate the measurements, two inductors $L_{c l}$ and $L_{c 2}$ that act as RF chokes are placed before the DC lines. The detailed parameters of these components are: $C_{1}=0.4 \mathrm{pF} ; C_{2}=100 \mathrm{pF} ; C_{3}=100 \mathrm{pF} ; R_{L}=5.1 \mathrm{k} \Omega$; and $L_{c l}=L_{c 2}=560 \mathrm{nH}$.

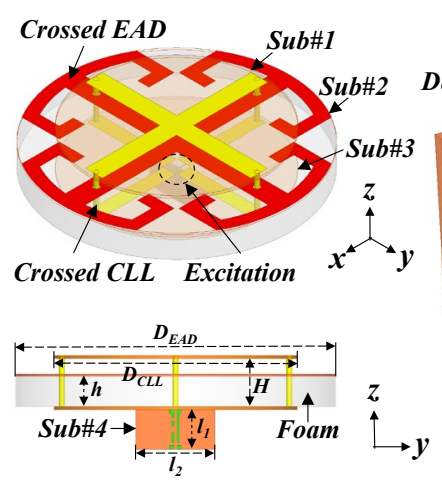

(a)

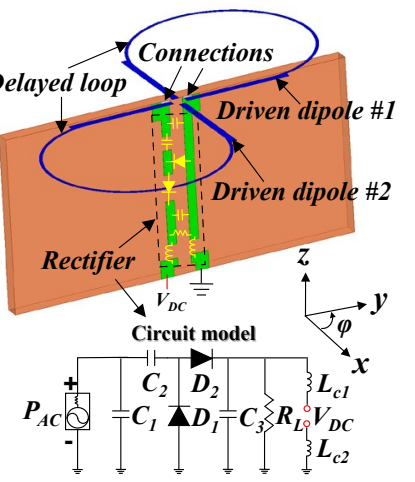

(b)
Fig. 8.The driven loop-based HCP rectenna configuration. (a) Perspective view (upper) and side view (below) of the entire system. (b) Perspective view of the vertical substrate $\# 4$ and the integrated rectifier, along with its circuit model.

As illustrated in Fig. 9, the power capture capacity of the developed system should exceed that of a rectenna based on a traditional half-wavelength dipole. Given the simulated half-power beamwidth values, very good wide-angle power capture performance was expected.

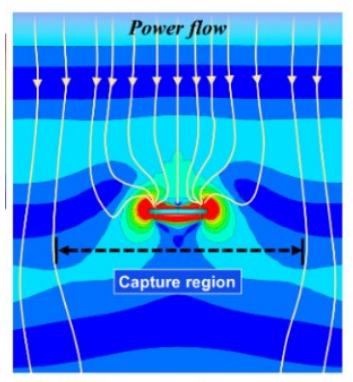

ES Huygens Rectenna

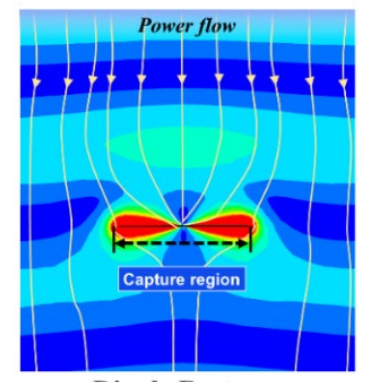

Dipole Rectenna
Fig. 9. Wireless power capture capacity comparison between the electrically small HCP rectenna and a half-wavelength LP dipole rectenna at the same operating frequency, $915 \mathrm{MHz}$ (Simulated with CST Microwave Studio).

\section{B. Fabricated prototype and measured results}

A driven loop-based HCP rectenna prototype was fabricated, assembled, and tested. The prototype was measured in an anechoic chamber as shown in Fig. 10(a). A broadband (800 MHz to $18 \mathrm{GHz}$ ) double-ridge LP horn antenna was used to emit the electromagnetic power to the rectenna. The realized gain of this horn antenna is $7 \mathrm{dBi}$ at $915 \mathrm{MHz}$. The horn antenna was connected to a signal generator from Keysight Technologies ${ }^{\mathrm{TM}}$ via a power amplifier type ZHL-20W-13SW+ from Mini-Circuits ${ }^{\mathrm{TM}}$ [43] and two coaxial cables. The gain of the amplifier is $50.5 \mathrm{~dB}$ and the total measured cable loss is $3 \mathrm{~dB}$. The rectenna prototype was placed 1.2 meters away from the horn aperture, which is in its far-field region, starting at $2 \mathrm{D}^{2} / \lambda_{0}=0.55$ $\mathrm{m}$ at $915 \mathrm{MHz}$. A multi-meter was connected to the output DC lines of the rectifier to measure the converted DC voltage. Since all the parameters are known, the power received by the rectenna was calculated with the classic Friis transmission equation [44]. 


$$
\frac{P_{r}}{P_{t}}=G_{t} \times G_{r} \times\left(\frac{\lambda_{0}}{4 \pi R}\right)^{2}
$$

The AC to DC conversion efficiency is determined by the ratio of the output DC power to the received AC power, $P_{r}$.

Photos of the rectifier in the prototype are shown in Fig. 10(b) and $10(\mathrm{c})$. The total length of the rectifier is only $1.0 \mathrm{~cm}$. The integration of the HCP antenna and the rectifier circuit is seamless. Consequently, even with the vertical Sub\#4, the entire system is very compact.

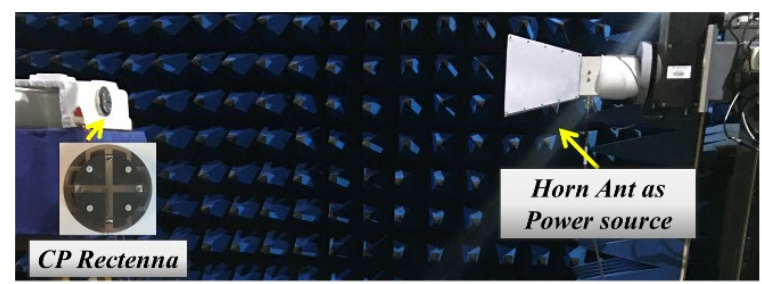

(a)

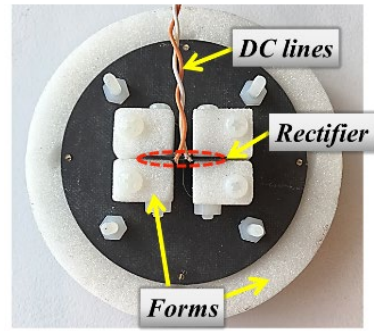

(b)

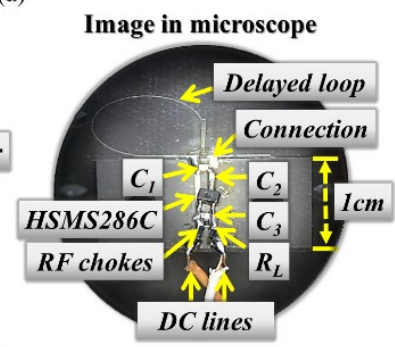

(c)
Fig. 10. Measurement setup and detailed prototype images. (a) AUT. (b) Bottom view of the assembled prototype. (c) Enlarged microscope view of the rectifier connected with the loop element.

Since the horn antenna does not generate a CP field but a LP one, it was rotated four times from $\varphi=0^{\circ}$ to $135^{\circ}$ in steps of $45^{\circ}$ to accommodate measuring the output DC voltage values in each LP state as functions of the source frequency from 900 to $930 \mathrm{MHz}$. The recorded values are shown in Fig. 11(a). The best overlap (smallest DC voltage differences between the four LP states) and, hence, the best CP power capture occurred at $904.5 \mathrm{MHz}\left(\lambda_{0}=331.44 \mathrm{~mm}\right)$, which is only a $1.1 \%$ shift away from the simulated value. This slight frequency shift is due to fabrication and assembly errors. At $904.5 \mathrm{MHz}$, the DC voltage values are $5.47,5.3,5.65$, and $5.9 \mathrm{~V}$, respectively, for the horn rotation angle $0^{\circ}, 45^{\circ}, 90^{\circ}$, and $135^{\circ}$. The measured voltage values at the simulated AR minimum frequency, 914.5 MHz, are plotted in Fig. 11(b)along with the simulated LP realized gain values of the HCP antenna in the broadside direction (i.e., $-3 \mathrm{~dB}$ from their $\mathrm{CP}$ values) as functions of the rotation angle $\varphi$. It is clearly seen that the measured DC voltages for each angle agree very well with their simulated LP values.

To further characterize the CP capture capacity, the prototype was rotated along the x-axis indicated in Fig. 10(a) and the output DC voltages were recorded. The measured values are $5.5 \mathrm{~V}, 5.17 \mathrm{~V}$ and $4.18 \mathrm{~V}$ when the HCP rectenna was rotated by $+30^{\circ},+45^{\circ}$, and $+60^{\circ}$ in clockwise manner as observed from the $+\mathrm{z}$-axis (top view). The values are $5.5 \mathrm{~V}, 5.1 \mathrm{~V}$ and $4.2 \mathrm{~V}$ when the rectenna was rotated $-30^{\circ},-45^{\circ}$, and $-60^{\circ}$ in anti-clockwise manner. The closeness of the values indicates that a broad-angle wireless power capture capacity was realized, confirming the simulated wide half-power beamwidth of the HCP antenna.

Finally, Fig. 12 shows the measured output DC voltage and the associated AC to DC conversion efficiency as functions of the captured power level. The peak measured efficiency is $90.6 \%$, which is very close to the simulated value, $88 \%$, and the maximum achievable value of the rectifier. Note that the measured value is actually slightly higher. This feature has been confirmed with simulations to be a consequence of the actual performance characteristics of the diodes. Moreover, the maximum measured value is $8.8 \%$ higher than the one attained in our original HCP rectenna work [31], which has an unbalanced crossed dipole feed structure. This increase is a direct result of the absence of the lossy inductor used in [31]. Although increasing the length of the driven dipole proved effective for impedance tuning in [45], this approach cannot be applied to modify the original HCP design [29] for matching to the rectifier. The stringent requirements faced herein for both the impedance and AR values cannot be satisfied simultaneously with that original driven crossed dipole design. Fortunately, as has been emphasized, this outcome can be facilitated by the driven loop-based HCP antenna design, which matches the HCP antenna directly to the rectifier.

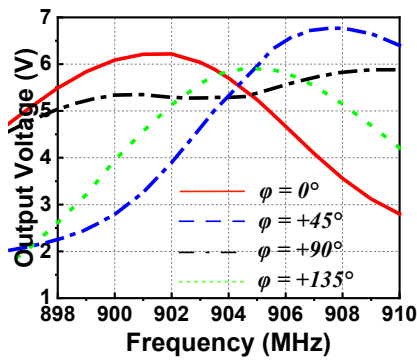

(a)

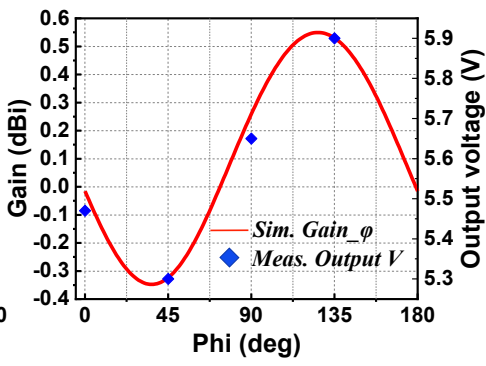

(b)
Fig. 11. Measured DC output voltage values as functions of the horn antenna rotation angle $\varphi$ (at $914.5 \mathrm{MHz}$ ) and the corresponding simulated broadside LP gain values, Gain_ $\varphi$.

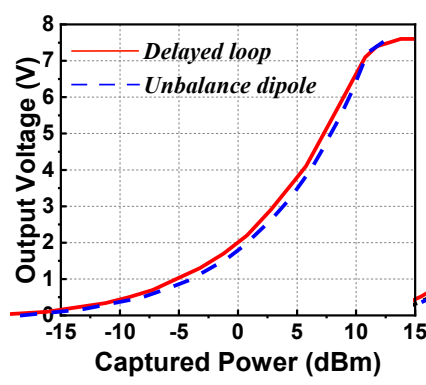

(a)

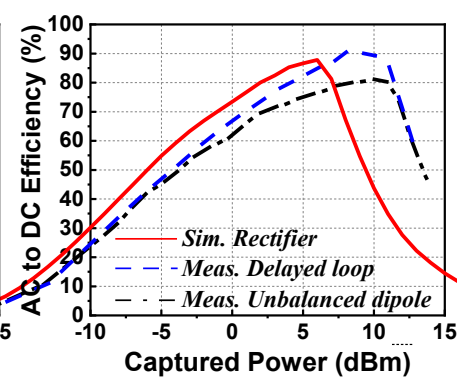

(b)
Fig. 12.HCP rectenna results as functions of the captured power at $904.5 \mathrm{MHz}$. (a) Measured output DC voltage. (b) Comparison of the measured and simulated AC to DC conversion efficiencies.

\section{CONCLUSION}

Two innovative driven dipole structures were introduced into the electrically small, NFRP HCP antenna of the original HCP rectenna in [31] to significantly enhance its conversion efficiency. It was demonstrated that the spiral line-based HCP antenna has a capacitive input impedance and, hence, is an ideal match to inductive external circuits. On the other hand, the driven loop-based HCP antenna has an inductive input impedance and, hence, is an ideal match for capacitive ones. Consequently, it was adapted to the HCP rectenna design and optimized to match its HCP antenna directly to its rectifier with no matching elements. Without the presence of the lossy matching inductor required in [31], the maximum achievable AC to DC conversion efficiency was reached. The measured value of fabricated prototype driven loop-based HCP rectenna has a measured maximum conversion efficiency of $90.6 \%$, which is $8.8 \%$ higher than the original $\mathrm{HCP}$ rectenna with its unbalanced dipole structure. Given its highly compact size $\left(0.12 \lambda_{0}\right.$ radius and $\left.k a \sim 0.77\right)$, wide angle power capture capacity, and excellent conversion performance, the system is an excellent candidate for future WPT-enabled IoT devices. 


\section{REFERENCES}

[1] A. Costanzo and D. Masotti, "Energizing 5G: Near- and far-field wireless energy and data transfer as an enabling technology for the 5G IoT," IEEE Microw. Mag., vol. 18, no. 3, pp. 125-136, May 2017.

[2] Z. Popovic, "Cut the cord: Low-power far-field wireless powering," IEEE Microw. Mag., vol. 14, no. 2, pp. 55-62, Mar. 2013.

[3] N. B. Carvalho, et al., "Wireless power transmission: R\&D activities within Europe," IEEE Trans. Microw. Theory Techn., vol. 62, no. 4, pp. 1031-1045, Apr. 2014.

[4] N. Shinohara, "Beam control technologies with a high-efficiency phased array for microwave power transmission in Japan," Proc. IEEE, vol. 101, no. 6, pp. 1448-1463, Jun. 2013.

[5] M. R. Palattella, et al., "Internet of things in the $5 \mathrm{G}$ era: Enablers, architecture, and business models," IEEE J. Sel. Area. Commun., vol. 34, no. 3, pp. 510-527, Mar. 2016.

[6] H. J. Visser and R. J. M. Vullers, "RF energy harvesting and transport for wireless sensor network applications: Principles and requirements," Proc. IEEE, vol. 101, no. 6, pp. 1410-1423, Jun. 2013.

[7] O. Bjorkqvist et al., "Wireless sensor network utilizing radio-frequency energy harvesting for smart building applications," IEEE Antennas Propag. Mag., vol. 60, no. 5, pp. $124-136$, Oct. 2018.

[8] S. Kim, et al., "Ambient RF energy-harvesting technologies for self-sustainable standalone wireless sensor platforms," Proc. IEEE, vol. 102, no. 11, pp. 1649-1666, Nov. 2014.

[9] K. Thiyagarajan, S. Kodagoda, R. Ranasinghe, D. Vitanage, and G. Iori, "Robust sensing suite for measuring temporal dynamics of surface temperature in sewers," Sci Rep., vol. 8, no. 1, 2018.

[10] K. Thiyagarajan, S. Kodagoda, L. V. Nguyen, and R. Ranasinghe, "Sensor failure detection and faulty data accommodation approach for instrumented wastewater infrastructures," IEEE Access, vol. 6, pp. 56 562-56 574, 2018.

[11] W. C. Brown, "The history of power transmission by radio waves,"IEEE Trans. Microw. Theory Techn., vol. MTT-32, no. 9, pp. 1230-1242, Sep. 1964.

[12] J. O. McSpadden, L. Fan, and K. Chang, "Design and experiments of a high conversion efficiency $5.8 \mathrm{GHz}$ rectenna", IEEE Trans. Microw. Theory Techn., vol.46, no.12, pp.2053-2060, Dec. 1998.

[13] A. Massa, G. Oliveri, F. Viani, and P. Rocca, "Array designs for long-distance wireless power transmission: State-of-the-art and innovative solutions," Proc. IEEE, vol. 101, no. 6, pp. 1464 - 1481, Jun. 2013.

[14] Z. Gu, S. Hemour, L. Guo, and K. Wu, "Integrated cooperative ambient power harvester collecting ubiquitous radio frequency and kinetic energy," IEEE Trans. Microw. Theory Techn., vol. 66, no. 9, pp. 4178-4190, Sep. 2018.

[15] C. Liu, Y. X. Guo, H. Sun, and S. Xiao, "Design and safety considerations of an implantable rectenna for far-field wireless power transfer," IEEE Trans. Antennas Propag., vol. 62, no. 11, pp. 5798-5806, Nov. 2014.

[16] H. Sun, Y. X. Guo, M. He, and Z. Zhong, "Design of a high-efficiency $2.45-\mathrm{GHz}$ rectenna for low-input-power energy harvesting," IEEE Antennas Wireless Propag. Lett., vol. 11, pp. 929-932, 2012.

[17] H. Sun and G. Wen, "A new rectenna using beamwidth-enhanced antenna array for RF power harvesting applications," IEEE Antennas Wirel. Propag. Lett., vol.16, pp. $1451-1454,2017$.

[18] M. Zeng, A. S. Andrenko, X. Liu, Z. Li, and H.-Z. Tan, "A compact fractal loop rectenna for RF energy harvesting," IEEE Antennas Wirel. Propag. Lett., vol.16., pp. $2424-2427,2017$.

[19] T. S. Almoneef, F. Erkmen, M. A. Alotaibi, and O. M. Ramahi, "A new approach to microwave rectennas using tightly coupled antennas," IEEE Trans. Antennas Propag., vol. 66, no. 4, pp. 1714 - 1724, Apr. 2018.

[20] S. Shen, C.-Y. Chiu, and R. D. Murch, "Multiport pixel rectenna for ambient RF energy harvesting," IEEE Trans. Antennas Propag., vol. 66, no. 2, pp. 644-656, Feb. 2018.

[21] V. Palazzi, et al., "A novel ultra-lightweight multiband rectenna on paper for RF energy harvesting in the next generation LTE bands," IEEE Trans. Microw. Theory Techn., vol. 66, no. 1, pp. 366-379, Jan. 2018.

[22] V. Palazzi, M. delPrete, and M. Fantuzzi, "Scavenging for energy: A rectenna design for wireless energy harvesting in UHF mobile Telephony Bands," IEEE Microw. Mag., vol. 18, no. 1, pp. 91-99, Jan.-Feb. 2017.

[23] C. Song, Y. Huang, P. Carter, J. Zhou, S. D. Joseph, and G. Li, "Novel compact and broadband frequency-selectable rectennas for a wide input-power and load impedance range," IEEE Trans. Antennas Propag., vol. 66, no. 7, pp. 3306 -3316, Jul. 2018.
[24] Z. Popović, E. A. Falkenstein, D. Costinett, and R. Zane, "Low-power far-field wireless powering for wireless sensors," Proc. IEEE, vol. 101, no. 6, pp. 1397-1409, Jun. 2013.

[25] M. Mattsson, C. I. Kolitsidas, and B. L. G. Jonsson, "Dual-band dual-polarized full-wave rectenna based on differential field sampling," IEEE Antennas Wirel. Propag. Lett., vol. 17, no. 6, pp. 956 - 959, Jun. 2018.

[26] B. Strassner and K. Chang, "5.8-GHz circularly polarized rectifying antenna for wireless microwave power transmission," IEEE Trans. Microw. Theory Techn., vol. 50, no. 8, pp. 1870-1876, Aug. 2002.

[27] Z. Harouni, L. Cirio, L. Osman, A. Gharsallah, and O. Picon, "A dual circularly polarized $2.45-\mathrm{GHz}$ rectenna for wireless power transmission," IEEE Antennas Wirel. Propag. Lett., vol. 10, pp. 306 - 309, Apr. 2011.

[28] C. Song, et al., "A novel six-band dual CP rectenna using improved impedance matching technique for ambient RF energy harvesting," IEEE Trans. Antennas Propag., vol. 64, no. 7, pp. 3160-3171, Jul. 2016.

[29] Y. Yang, et al., "A $5.8 \mathrm{GHz}$ circularly polarized rectenna with harmonic suppression and rectenna array for wireless power transfer," IEEE Antennas Wirel. Propag. Lett., vol. 17, no. 7, pp. 1276 - 1280, Jul. 2018.

[30] Y. Liu, K. Huang, Y. Yang, and B. Zhang, "A low-profile lightweight circularly polarized rectenna array based on coplanar waveguide," IEEE Antennas Wirel. Propag. Lett., vol. 17, no. 9, pp. 1659-1663, Sep. 2018.

[31] W. Lin and R. W. Ziolkowski, "Electrically small, low profile, highly efficient, Huygens dipole rectennas for wirelessly powering Internet-of-Things (IoT) devices," IEEE Trans. Antennas Propag., vol. 67, no. 6, pp. 3670-3679, June. 2019.

[32] C. R. Valenta and G. D. Durgin, "Harvesting wireless power: Survey of energy-harvester conversion efficiency in far-field, wireless power transfer systems," IEEE Microw. Mag., vol. 15, no. 4, pp. 108-120, Jun. 2014.

[33] W. Lin and R. W. Ziolkowski, "Electrically-small, low-profile, Huygens circularly polarized antenna," IEEE Trans. Antennas Propag., vol. 66, no. 2, pp. 636-643, Feb. 2018.

[34] T. Ohira, "Power efficiency and optimum load formulas on RF rectifiers featuring flow-angle equations," IEICE Electron. Express, vol. 10, no. 11, pp. 1-9, Jun. 2013.

[35] S. X. Ta, I. Park, and R. W. Ziolkowski, "Crossed dipole antennas: a review.," IEEE Antennas Propag. Mag., vol. 57, no. 5, pp. 107-122, Oct. 2015.

[36] Y. He, W. He, and H. Wong, "A wideband circularly polarized cross-dipole antenna," IEEE Antenna Wireless Propag. Lett., vol. 13, pp. 67-70, 2014.

[37] W. Lin, S. L. Chen, R. W. Ziolkowski, and Y. J. Guo, "Reconfigurable, wideband, low-profile, circularly polarized antenna and array enabled by an artificial magnetic conductor ground," IEEE Antennas Propag. Mag., vol. 66, no. 3, pp. 1564-1569, Mar. 2018.

[38] Data Sheet of HSMS-286x Series Schottky Diodes, Broadcom Limited, Application Note [Online]. Available: https://www.broadcom.com.

[39] N. Zhu, R. W. Ziolkowski, and H. Xin, "A metamaterial-inspired, electrically small rectenna for high-efficiency low power harvesting and scavenging at the GPS L1 frequency," Appl. Phys. Lett., vol. 99, 114101, Sep. 2011.

[40] Q. W. Lin and X. Y. Zhang, "Differential rectifier using resistance compression network for improving efficiency over extended input power range," IEEE Trans. Microw. Theory Techn., vol. 64, no. 9, pp. 2943-2954, Sep. 2016.

[41] Z. X. Du and X. Y. Zhang, "High-efficiency single- and dual-band rectifiers using a complex impedance compression network for wireless power transfer," IEEE Trans. Industrial Electron., vol. 65, no. 6, pp. 5012-5022, June 2018.

[42] N. Shariati, W. ST Rowe, J. R. Scott, and K. Ghorbani, "Multi-service highly sensitive rectifier for enhanced RF energy scavenging." Sci. Rep., vol. 5, 9655, May 2015.

[43] Coaxial high power amplifier ZHL-20W-13+ Datasheet. [Online] Available: https://www.minicircuits.com/pdfs/ZHL-20W-13+.pdf, Jun. 2018.

[44] C. A. Balanis, 3rd Ed., Antenna Theory. New York: John Wiley \& Sons, 2005.

[45] W. Lin and R. W. Ziolkowski, "Electrically small Huygens antenna-based fully-integrated wireless power transfer and communication system," IEEE Access, vol. 7, pp. 39762-39769, Mar. 2019. 